\title{
Variations
}

Variations

Revue internationale de théorie critique

$17 \mid 2012$

Critique du travail

\section{Le courage comme vertu cardinale du sujet politique}

\section{Manuel Cervera-Marzal}

\section{(2) OpenEdition}

Journals

Édition électronique

URL : http://journals.openedition.org/variations/364

DOI : 10.4000/variations.364

ISSN : 1968-3960

Éditeur

Les amis de Variations

Référence électronique

Manuel Cervera-Marzal, «Le courage comme vertu cardinale du sujet politique », Variations [En ligne], 17 | 2012, mis en ligne le 15 octobre 2012, consulté le 19 avril 2019. URL : http://

journals.openedition.org/variations/364; DOI : 10.4000/variations.364

Ce document a été généré automatiquement le 19 avril 2019

Les ami•e•s de Variations 


\title{
Le courage comme vertu cardinale du sujet politique
}

\author{
Manuel Cervera-Marzal
}

1 Les mots ont une importance, jusque dans leur dernière syllabe. Aborder la question du sujet politique par l'angle de la subjectivation plutôt que par celui de la subjectivité n'est pas anodin. La notion de subjectivation implique de penser le sujet comme un processus, une progression, une réalisation toujours en cours, et non un donné, un statut, un état déjà atteint et achevé. Le sujet politique est en construction permanente. Le citoyen n'est pas un produit dont on pourrait constater l'existence mais un projet qu'il s'agit justement de produire.

2 Dès lors se pose la question essentielle des modalités de la subjectivation politique: comment produire de la citoyenneté ${ }^{1}$ ? quelle voie emprunter pour faire advenir un sujet politique? La question doit être mieux formulée: il ne s'agit pas de «produire» ou de «faire advenir" un citoyen par un mécanisme de fabrication qui serait extérieur à l'individu. C'est au contraire à chacun qu'il revient de s'élever par lui-même de la condition d'animal social à celle d'animal politique, de la condition de sujet-assujetti à celle de sujet-souverain, d'animal laborans à zoon politikon. L'enjeu est alors de localiser le lieu de passage du stade pré-politique à celui politique. Une manière de résoudre cette question et d'identifier le processus par lequel un individu engage par ses propres soins le processus de subjectivation politique consiste à se demander ce qui différencie un sujet politique d'un sujet pré-politique. Autrement dit, quelle est la vertu citoyenne par excellence? A l'instar d'Hannah Arendt et de toute une tradition philosophique ayant choisi de penser le politique à partir de l'action, nous soutenons que le courage est la première de toutes les vertus politiques.

3 La question de la subjectivation politique nous conduit alors à analyser la notion de courage, entendue au sens politique, en tant que vertu cardinale du citoyen et qu'élément discriminant entre les citoyens et les non-citoyens. Toujours en nous appuyant sur une philosophie politique de l'action, nous pouvons définir le courage comme ce qui fait passer un individu de l'intelligence d'un problème à la volonté de le résoudre. Car comme l'écrit 
Thoreau dans La désobéissance civile, "Il y a des milliers de gens qui par principe s'opposent à l'esclavage et à la guerre mais qui en pratique ne font rien pour y mettre un terme. [...] Il y a 999 défenseurs de la vertu pour un seul homme vertueux $»^{2}$. Le courage est alors ce qui comble la distance entre la compréhension d'un problème et la décision d'agir pour y mettre un terme. Il sépare la condamnation verbale d'une injustice de l'engagement actif contre cette injustice. Un possible obstacle à l'action réside dans le fait que « les gens sont pragmatiques. Ils veulent le changement, mais ils se sentent désarmés, seuls, et craignent d'être le brin d'herbe qui dépasse et sera donc fauché le premier. Ils attendent quelqu'un qui fera le premier mouvement $»^{3}$. Le citoyen est cet homme du "premier mouvement ", celui qui parie que s'il fait le premier pas, d'autres le suivront suffisamment vite pour empêcher qu'ils ne soient fauchés.

Le citoyen est celui qui a le courage de transformer ses paroles en actes, ses pensées en engagement, sa position intellectuelle en conviction réelle. Bref, celui qui passe de l'indignation à la mobilisation. Lorsque nous disons ici que le courage consiste à passer de la parole aux actes, nous avons conscience que certaines formes de paroles sont déjà des actes et donc que certaines paroles sont courageuses : par exemple, le fait de dénoncer publiquement une injustice protégée par l'omerta. Ce qu'on appelle donc ici « le passage de la parole aux actes » désigne le passage d'une parole timorée qui ne s'exprime que dans les cercles restreints de la sphère privée à une parole politique qui affirme ouvertement et publiquement ce qu'elle a à dénoncer ${ }^{4}$. De même, l'action politique peut être une pensée, car il y a des pensées qui sont si radicales et affranchies des normes établies qu'elles en sont courageuses.

Le courage, invitant ainsi à l'action, nous fait pénétrer dans l'espace public. Et c'est bien le propre du citoyen que d'inscrire son action dans cet espace public, de libérer ses convictions de la prison de sa pensée pour les laisser s'exposer à tous et s'incarner en actes. Le sujet politique fait preuve de courage en sortant des coulisses pour passer sur le devant de la scène politique. Frédéric Gros a vu juste lorsqu'il écrit que « le courage est surtout de s'exposer au sens où il n'y a de vrai courage qu'extériorisé $»^{5}$. Le courage est une vertu extérieure, mais l'extériorité ne doit pas ici être entendue péjorativement comme superficialité ou apparence trompeuse. Le courage est extérieur au sens où comme intention il ne vaut rien, puisqu'il est précisément actualisation et réalisation de l'intention. Il se démontre dans la confrontation au réel. Lorsqu'il se cantonne au verbal il n'est que lâcheté. « Les courageux en paroles, on les connaît : ce sont les plus lâches sur le terrain. Les braves laissent les autres parler et se taisent $»^{6}$.

6 Enfin, faut-il le rappeler, le courage s'exerce toujours dans le cadre d'une certaine gratuité . Le citoyen qui fait preuve de bravoure ne saurait attendre pour son geste la moindre récompense ou gratification. Il ne saurait pas davantage trouver la motivation de son acte dans une rétribution financière. Car dans ce cas l'idéal du courage serait terni à jamais et il faudrait plutôt parler d'un homme zélé ou d'un employé dévoué que d'un citoyen courageux.

7 Cette définition générale du courage étant posée, il nous semble nécessaire d'aller plus loin. Le courage n'est pas une notion monolithique et unifiée. En effet, la modernité politique, tant au niveau de la pratique que des idées, nous permet de dresser une typologie tripartite des différentes formes de courage: le courage de la vérité, qu'à l'instar de Foucault nous nommerons parrhèsia; le courage de la liberté, qu'à l'instar d'Arendt nous nommerons héroïsme; et le courage de la non-violence, qu'à l'instar de Gandhi nous nommerons satyagraha. La parrhèsia est le courage de dire le vrai dans un 
monde de mensonges et de "faire de sa vie un témoignage de vérité ». L'héroïsme est le courage de préférer la mort à la servitude au sens où, comme le disait le révolutionnaire mexicain Emiliano Zapata, « mieux vaut mourir debout que de vivre à genoux ! » Enfin, le satyagraha est le courage de « combattre le mal tout en refusant d'imiter sa violence ».

À chacune de ces formes de courage correspond une figure de la subjectivation politique : le militant fait preuve du courage de la vérité, le révolutionnaire démontre son courage de la liberté et le désobéissant manifeste le courage de la non-violence. Pour mieux illustrer les catégories que nous élaborons ici, nous nous référerons respectivement à trois personnages historiques : Simone Weil en tant qu'archétype du militant (vérité), George Orwell comme modèle du révolutionnaire (liberté), et Mohandas K. Gandhi comme représentant du désobéissant (non-violence).

\section{La parrhèsia politique : militer pour le vrai en faisant de sa vie un « témoignage de vérité »}

Dans ses derniers cours au Collège de France, en 1983 et 1984, Michel Foucault revient à plusieurs reprises sur une forme particulière d'existence qui, à l'instar des cyniques grecs ${ }^{7}$, consiste à faire de sa vie un scandale et un plaidoyer pour la vérité. Nous avons tous en mémoire Diogène, se promenant dans son tonneau, se masturbant en public, et recevant le roi Alexandre, venu lui rendre visite, en le traitant de bâtard. Il s'agissait par ce comportement outrageant d'adopter une posture éthique faisant de sa vie un «témoignage de vérité ». Par ce biais, Foucault interroge la fonction du "dire-vrai » ( parrhèsia) en politique. La thèse est la suivante : la parrhèsia - entendue comme courage de dire la vérité - et la démocratie entretiennent depuis toujours un lien intime. Dans son cours du 2 février 1983, Foucault explique que la démocratie athénienne repose non sur deux, comme on l'a souvent répété, mais sur trois principes. L'isonomia, bien sûr, c'est-àdire la loi égale pour tous et l'égalité de tous devant la loi. L'isegoria, aussi, qui désigne l'égale liberté de parole : quelle que soit son métier et sa position sociale, chaque citoyen est libre d'exprimer son opinion à l'assemblée. Mais à ce régime de la "parole démocratique ", Foucault ajoute une seconde dimension : la parole libre et égale (isegoria) se voit doubler de la "parole vraie», du franc-parler, du courage d'exprimer publiquement ce qui dérange, qui ne fait pas plaisir à entendre mais doit être dit. La parrhèsia se définit alors comme la prise de parole d'un homme courageux qui, refusant la complaisance et la flatterie, décide de dire au peuple la vérité, quel que soit le prix qu'il en coûte (pour le peuple, mais surtout pour l'homme qui s'adresse au peuple).

Le style de vie de l'homme courageux, n'hésitant pas à prendre la parole pour faire entendre la vérité, a perduré au cours de l'histoire. L'ascétisme chrétien médiéval ${ }^{8}$ a été, selon Foucault, le véhicule qui a transmis des Anciens aux Modernes ce mode d'existence initialement mis au point par les cyniques. Ainsi, la modernité occidentale offre à notre étude une figure archétypique du courage de la vérité : le militant politique. Nous prenons ici nos distances avec Foucault qui identifie à tort, nous semble-t-il, le militant et le révolutionnaire. En effet, selon nous, le militant est celui qui rappelle à l'ordre le révolutionnaire, en lui indiquant qu'il se contente de trop peu et ne s'investit pas suffisamment pour la cause de la vérité. Le militant est celui qui, à l'instar de Simone Weil, ne dénonce pas seulement l'abomination du capitalisme impérialiste et la barbarie des franquistes. Cette vérité est trop facile à dire. Cette dénonciation est si évidente que 
l'expliciter ne fait pas avancer la vérité. Ils furent des millions de révolutionnaires de tout poils (anarchistes, trotskistes, communistes) à être conscients de l'alliance entre «fascisme et grand capital $»^{9}$ et à combattre leurs effets barbares. Non, la vérité qu'affirme le militant est autrement plus profonde. Cette vérité exige un réel courage car elle concerne et atteint le propre camp du militant, elle s'adresse à ses amis les plus proches. Cette vérité est autocritique. Elle est, comme l'écrit Simone Weil, une « réflexion pour déplaire $»^{10}$. Et c'est pourquoi même parmi les siens, surtout parmi les siens, le militant est mal vu. Il sacrifie sa respectabilité et sa réputation sur l'autel de la vérité et de la justice et par ce geste de bravoure il s'expose directement à l'exclusion et à la marginalisation. Il est stigmatisé comme traître alors qu'il est précisément le plus fidèle à la cause en étant le plus critique envers les armes mises en œuvre pour cette cause. Le militant, par son attachement acharné à la vérité, ne cesse jamais de perturber la bonne conscience du révolutionnaire qui, s'étant positionné du côté des dominés, se croit désormais épargné de toute critique.

11 Le militant, qui éclaire courageusement le révolutionnaire sur l'insuffisance de son engagement pour la vérité, ne doit pas non plus être identifié à la figure du partisan. Ce dernier est celui qui, croyant faire œuvre de militantisme, souhaite démontrer son amour de la vérité en s'engageant au sein du parti politique qui en est à ses yeux dépositaire. Or nous devons distinguer radicalement l'esprit militant de l'esprit partisan. La différence réside dans la manière de penser les problèmes politiques et dans la façon de réfléchir en général. L'un comme l'autre orientent leur raisonnement en direction de la vérité; mais alors que le militant s'achemine correctement dans l'univers de la pensée, le partisan avance comme un boiteux aux yeux bandés. Le problème avec les partisans, écrit Simone Weil, est qu'« on en est arrivé à ne presque plus penser, dans aucun domaine, qu'en prenant position "pour " ou "contre » une opinion. Ensuite on cherche des arguments, selon le cas, soit pour, soit contre $»^{11}$. C'est là l'exact résultat de l'adhésion à un parti. Or il $\mathrm{y}$ a dans cet esprit partisan deux erreurs intellectuelles. La première consiste à prendre position d'abord, et à ensuite chercher à justifier sa position. Or une investigation rationnelle, réellement soucieuse de la vérité, doit procéder à l'inverse. Le tâche préliminaire consiste à récolter, sélectionner, trier et organiser les éléments d'information. C'est seulement après avoir fait ce travail qu'il devient possible de se former une opinion rationnelle, conforme (autant qu'il est humainement possible) à la vérité. Ainsi presque partout l'opération de prendre parti, de prendre position pour ou contre, s'est substituée à l'opération de la pensée. La seconde tare de l'esprit partisan est qu'en abordant systématiquement les problèmes sur un mode binaire (pour/contre) on s'empêche de les envisager dans toute leur complexité, qui requiert souvent l'élaboration d'un dépassement par une troisième voie. Aussi faut-il bien comprendre que la parrhèsia n'est pas qu'un courage en acte (incarner la vérité à la manière des cyniques grecs) ou en parole (dire publiquement la vérité qui dérange) : elle est aussi un courage en pensée. Il s'agit alors de faire preuve de courage intellectuel en refusant de verser dans le simplisme de l'esprit partisan. Réfléchir à la manière d'un militant, c'est-à-dire en chercheur aguerri de la vérité, n'est pas une tâche pour les lâches. Cela demande un effort dont seul le militant courageux est capable. Il est facile et tentant de faire l'économie de la réflexion en adhérant sans critique aux positions que notre parti aura définies pour nous. Car, comme l'écrit Simone Weil, «il n'y a rien de plus confortable que de ne pas penser ${ }^{12}$ ! Rien de plus lâche, ajouterons-nous. 
12 Ayant ainsi dressé à grands traits la figure du militant et de la parrhèsia dont il fait preuve, nous devons préciser que le courage de la vérité n'est pas nécessairement la vérité du courage. Autrement dit, la modernité politique propose d'autres variantes du courage, dont la première est celle de l'héroïsme, courage de la liberté.

\section{L'héroïsme du révolutionnaire : « plutôt mourir debout que vivre à genoux »}

13 L'héroïsme théorisé par Arendt, notamment dans Qu'est-ce que la politique?, se fonde sur le courage et l'amour de la liberté de celui qui préfère la mort à la servitude. Ainsi, comme l'écrit la philosophe allemande, «seul peut être libre celui qui est prêt à risquer sa vie »13. Savoir dépasser la peur de la mort pour pénétrer l'espace public, c'est faire preuve de courage, «la première de toutes les vertus politiques »" Le héros, au nom de la liberté, brave sa peur de la mort et ose exposer publiquement son corps malgré les risques que cela comporte pour sa vie. Ce passage des pénombres de la sphère privée aux projecteurs de l'espace public s'opère de manière soudaine, à la façon d'un bond, d'un grand saut en avant $^{15}$.

Ce courage de la liberté, qui prit naissance dans la polis grecque, concerne aussi le révolutionnaire des temps modernes. Mais qui parle de révolution est aujourd'hui suspect des pires atrocités. Dès lors, on ne s'étonne pas que certains expriment leurs craintes visà-vis du penchant totalitaire du héros arendtien. "Le XXe siècle, reconnaît Miguel Abensour, a connu des formes d'héroïsme totalitaire, soit dans l'exaltation du soldat en proie à la mobilisation totale, soit dans la glorification de l'homme nouveau ${ }^{16}$. Mais il faut aussitôt préciser que le héros d'Arendt, loin de menacer le politique (comme le fait totalitarisme, qui est destruction et non excès du politique), se révèle dans et pour la polis. C'est cette qualité politique de l'héroïsme qui l'empêche de glisser vers la tyrannie. Car c'est bien le propre de certaines formes d'héroïsme, par exemple esthétique ou métaphysique, que de renforcer la prémisse inégalitaire et de favoriser la domination d'une minorité sur le plus grand nombre. Mais chez Arendt, le caractère politique de l'héroïsme signifie que ce dernier se déploie dans les limites de la condition humaine. Le héros n'ayant rien d'un surhomme, nous sommes donc face à un héroïsme radicalement nouveau : un héroïsme à la portée de tous. Le courage de la liberté, parce que la liberté est un désir logé dans la nature humaine et donc dans chacun d'entre nous, est un héroïsme du « n'importe qui », au sens de Rancière. Ainsi Arendt réconcilie héroïsme et démocratie. Précisons enfin que cet héroïsme, en plus d'être accessible à tous, se manifeste à plusieurs, dans l'agire di concerto, expérience de pluralité. La subjectivation politique est toujours celle d'un «nous", jamais celle d'un «je». En cela, l'héroïsme des révolutionnaires est l'exact opposé de celui solitaire d'un De Gaulle ou d'un James Bond. Il s'agit d'une forme de courage nouvelle et paradoxale que, selon l'heureuse formule de Miguel Abensour, nous pourrions nommer « héroïsme des sans-nom ». Car, comme l'ont remarqué leurs contemporains et après eux les historiens, « qui connaît ces héros qui en 1848 ou en 1871 prirent l'initiative d'un assaut ou résistèrent jusqu'à la mort sur les barricades, face aux forces de la répression ? ${ }^{17}$

Mais cette présentation quelque peu idyllique de l'héroïsme révolutionnaire ne doit pas en occulter son versant problématique. En faisant primer la liberté sur la vie humaine, car c'est bien de cela qu'il s'agit dans la maxime " plutôt mourir debout que vivre à genoux ", 
le révolutionnaire pénètre un espace instable où il risque à chaque instant de glisser vers le nihilisme. Le révolutionnaire est prêt, s'il le faut, à sacrifier sa vie, parfois même celle d'autrui, pour sauvegarder la liberté. Une telle disposition n'est pas forcément souhaitable car, explique Albert Camus, c'est adopter une conception absolutiste de la liberté, selon laquelle si la liberté n'est pas totale la vie ne vaut pas d'être vécue. Or, la liberté absolue, ajoute Camus, « c'est le droit pour le plus fort de dominer $»^{18}$. La liberté la plus extrême signe l'arrêt de mort de la justice.

Cette recherche de la «consécration exclusive » de la liberté, Camus la nomme le «Tout ou Rien ». Le héros révolutionnaire qui adhère au « Tout ou Rien » se fourvoie. Il trahit sa vocation originelle. Se battant pour une liberté totale, illimitée, qui n'admet aucune frontière, pas même la liberté d'autrui, il croit faire preuve de courage, alors qu'il ne démontre que sa folie. L'esprit révolutionnaire, s'il veut atteindre son but, doit proposer une version relative et restreinte de la liberté. En effet, et l'on retrouve ici Arendt, l'héroïsme n'est le bienvenu que quand il est humain, c'est-à-dire lorsqu'il reconnaît sa finitude. Seul un courage mesuré peut être authentique. Autrement, nous sommes dans un simulacre de courage, une forme de martyr ou de sacrifice. S'il oublie ce principe de limitation, le héros bascule dans la logique du «Tout ou Rien » et aboutit à un nihilisme destructeur. Pour éviter ce travers, la révolution doit fonder une philosophie des limites et du relatif. Il importe de maintenir une tension permanente entre des couples notionnels dont il ne faut absolutiser aucun des deux éléments au détriment de l'autre. Il s'agit de sauvegarder l'équilibre précaire entre le refus et le consentement, la singularité et l'universel, l'individu et l'histoire et, surtout, la liberté et la vie. C'est au prix de cet effort intellectuel de chaque instant que le révolutionnaire évitera de se transformer en tortionnaire.

Il ne faut pas s'y tromper, le véritable héros ne méprise pas la vie. Il a beau la sacrifier pour la liberté, il la chérit comme ce qu'il a de plus précieux. Il n'est en rien louable d'offrir à un ami un objet qui nous appartient mais nous dégoûte. De même, il n'y a aucun mérite à donner sa vie lorsqu'on ne l'aime pas. Au contraire, il est d'autant plus courageux d'exposer sa vie qu'on y tient davantage ${ }^{19}$. Ce qui est admirable dans le courage, c'est précisément qu'on renonce à ce pour quoi l'on se bat: la vie humaine. Ajoutons que le héros n'est pas celui qui n'a pas peur, mais celui qui sait reconnaitre sa crainte de la mort tout en la dépassant et en trouvant les ressources mentales pour voir au-delà, pour comprendre que sa mort a un sens en tant qu'elle fait signe vers la liberté. Ainsi l'héroïsme révolutionnaire n'est pas de n'avoir jamais peur (tel est le propre du confiant et du naïf) mais d'affronter sa peur (tel est le propre de l'intrépide qui, étymologiquement, ne tremble pas devant le danger). Il y a là une exigence qui relève davantage de la force d'âme que de la force physique : la maîtrise de soi. Cette maîtrise s'exerce à plusieurs niveaux (dompter nos peurs et nos passions, réduire nos colères et nos faiblesses) et demande un apprentissage. Le contrôle de soi ne s'acquiert que par la persévérance et l'application répétée d'un certain nombre d'exercices. "Dorothée de Gaza recommande de commencer par s'habituer à retrancher de petites choses, une vaine curiosité, une parole inutile, pour se préparer peu à peu à de plus grands sacrifices $»^{20}$. Au terme d'une longue "thérapeutique des passions ", le héros, maître de soi, est apte à se battre pour la liberté au péril de sa vie.

18 Nous voudrions rappeler qu'il faut nous déshabituer à penser le héros comme un grand homme ou comme un être extraordinaire, mythologique, mi-dieu mi-homme. Le terme, tel que nous venons de le reconceptualiser, désigne avant tout ces héros anonymes qui, 
des sans-culottes aux zapatistes, ont fait vivre les révolutions modernes. Aussi, pour donner corps à cette figure et finalement poser paradoxalement un nom sur cet "héroïsme des sans-nom», nous voudrions conclure sur l'histoire de George Orwell. Lorsque durant la guerre civile espagnole Orwell s'engage dans les milices du POUM ${ }^{21}$, il n'a pas encore accédé à la célébrité. Aux yeux de ses camarades, il est leur égal, Eric Blair de son vrai nom, un nom parmi tant d'autres. Et c'est parce qu'il est encore anonyme que son expérience espagnole nous intéresse. Anglais d'origine indienne, rien ne le prédestinait à porter le fusil dans la Catalogne de 1936, si ce n'est sa conversion au "socialisme démocratique " entamée au contact des mineurs britanniques. Rien ne le prédestinait, rien ne l'y obligeait non plus. D'origine bourgeoise et sans lien aucun avec l'Espagne, il eut été facilement compréhensible qu'Orwell préfère conserver son emploi de libraire et rester vivre paisiblement à Londres. Mais s'il a choisi l'inconfort de la guerre et le risque de la mort, c'est bien qu'une force le poussait à agir et qu'une cause le pressait à s'engager : la liberté. Comme il l'écrit lui-même dans Hommage à la Catalogne, il n'était venu en Espagne que dans l'intention d'écrire quelques articles. Mais une fois arrivé sur place, constatant que les anarchistes avaient toujours la haute main sur la Catalogne et que la révolution battait de son plein, il lui parût «inconcevable de pouvoir agir autrement » qu'en s'engageant dans les milices ${ }^{22}$. À deux reprises, il faillit y laisser la vie. Mais l'intensité de cette menace ne suffit pas à faire renoncer le révolutionnaire qui grondait en lui. Il régnait à Barcelone et dans le cœur d'Orwell « la foi dans la révolution et [...] l'impression d'avoir soudain débouché sur une ère d'égalité et de liberté $»^{23}$. Cette idée d'une société sans classes était pour Orwell une cause méritant largement qu'on vienne "risquer sa peau pour elle ${ }^{24}$. Ceci étant dit, il nous semble que l'expérience catalane d'Orwell constitue un exemple représentatif de ce que nous désignons par « courage de la liberté ». L'Hommage à la Catalogne doit évidemment être lu avec un regard d'autant plus critique qu'il s'agit d'une autobiographie, mais l'on trouve dans ce récit l'ensemble des composantes qui caractérisent la figure du héros révolutionnaire.

\section{Le satyagraha, courage de la non-violence : « résister au mal autrement que par la violence »}

19 La non-violence n'est pas un refus du conflit mais la volonté d'y participer autrement que par la violence. Partant, ce que nous désignons par « courage de la non-violence » est la force de résister tout en refusant d'imiter la violence de l'adversaire. Il y a quelque chose de surprenant dans l'émergence de phénomènes de résistance non-violente ou de désobéissance civile. Expliquons-nous. Lutter sans armes contre un adversaire armé ne va pas de soi. Face à l'agresseur (que ce soit un occupant, un dictateur, ou autre), l'agressé dispose de trois options: la collaboration, la passivité, ou la résistance. La troisième option est la plus périlleuse. La résistance, une fois que l'on a choisi de s'y engager, peut être menée selon deux modalités : avec des armes, ou sans armes. Ceux qui résistent sans armes (les désobéissants) le font souvent faute de mieux, parce qu'ils n'ont tout simplement pas de fusils à disposition, et non par conviction morale. Mais peu importe, l'incompréhensible réside ici : comment un groupe d'individus désarmés peut-il décider d'entrer en résistance ou en action contre un adversaire qui, lui, dispose des armes et n'hésitera pas, s'il le faut, à en faire usage ? Un tel engagement exige un double courage : celui de dire "non", de résister; et celui de résister sans armes, c'est-à-dire en ayant conscience de la dissymétrie des moyens qui les oppose à leur adversaire. 
20 Le courage n'est pas une dimension optionnelle de la non-violence. Au contraire, il faut insister sur le fait que la non-violence, au sens où elle est une technique de lutte, ne saurait fonctionner sans le courage qui lui est propre. Pourquoi ? Il est immoral de chercher à résoudre un conflit par la force physique. Gandhi l'a toujours répété, «l'objectif du désobéissant consiste à convertir, non à contraindre, celui qui fait le mal » ${ }^{25}$ . Mais l'expérience a enseigné à Gandhi que la persuasion par la raison échoue souvent. Nous pouvons le constater au quotidien. Dans ces conditions, comment convaincre l'adversaire politique qu'il est dans l'erreur? Quand l'appel à la raison échoue, quel autre mécanisme permet la persuasion ? Gandhi répond : « l'appel au cœur ». " Pour obtenir un résultat décisif, explique-t-il, il ne suffit pas de convaincre la raison; il faut également toucher le cœur $»^{26}$. L'appel au cœur fonctionne comme une alternative ou un pas de plus à l'appel à la raison. Quand la tentative de persuasion par le discours rationnel a échoué, les opposants peuvent tenter d'utiliser des moyens de persuasion non-rationnels.

21 Il en découle la question suivante : comment l'appel au cœur peut convertir celui qui est dans l'erreur alors même que la raison a échoué ? Par la souffrance personnelle que s'inflige l'innocent, répond Gandhi. En acceptant volontairement de souffrir pour sa position morale, le désobéissant démontre sa force de conviction et dépasse le simple seuil du discours rationnel, qui n'exige aucun courage particulier. Il faut se sacrifier soimême pour toucher le cœur de l'adversaire et éveiller sa conscience endormie. Selon Gandhi, à celui qui me frappe je dois, comme Jésus l'a enseigné, tendre l'autre joue. Il se peut, explique Jean-Marie Muller, «que le fait de refuser d'imiter la violence de l'adversaire le surprenne, alors qu'il pensait mériter une réplique, le décontenance, le déconcerte, et finalement le désarme ${ }^{27}$. Car, note Gandhi, «il n'y a pas de satisfaction à tuer celui qui fait bon accueil à la mort et c'est pourquoi les soldats aiment attaquer l'ennemi lorsqu'il répond aux coups par des coups et à la violence par la violence ${ }^{28}$. Il ajoute: "Je cherche à émousser complètement l'épée du tyran, non pas en le heurtant avec un acier mieux effilé, mais en trompant son attente de me voir lui offrir une résistance physique. Il trouvera chez moi une résistance de l'âme qui échappera à son étreinte. Cette résistance d'abord l'aveuglera et ensuite l'obligera à s'incliner ${ }^{29}$. Celui qui offre sa personne en sacrifice désarme ainsi toute hostilité. La souffrance a le pouvoir de convertir les raisons les plus sourdes et les cœurs les plus endurcis ${ }^{30}$. Ainsi, le courage de la non-violence est une condition d'effectivité du satyagraha (action non-violente). Pour réussir à persuader l'adversaire qu'il se trouve dans l'erreur, le désobéissant doit courageusement s'opposer sans violence. La souffrance innocente du désobéissant permet de convertir l'adversaire plutôt que de le contraindre.

Il est utile ici de rappeler les mots sévères d'Arendt contre cette idée de Gandhi que le sacrifice de soi serait une preuve de la force de notre conviction et un témoignage de courage : «Il est regrettable que tant de personnes soient persuadées que la "volonté de se sacrifier soi-même" constitue la meilleure preuve de "l'intensité de l'engagement" et du sérieux de "celui qui désobéit par fidélité à une loi", car cette forme de fanatisme uniquement tendue vers son objectif est en général le fait d'excentriques et, de toute façon, a pour effet de rendre impossible une discussion rationnelle des données du problème $»^{31}$. Dans la même veine, Max Weber et l'Eglise catholique font remarquer que l'éthique du Sermon sur la montagne ne vaut que dans les relations entre individus, mais pas entre groupes et nations. L'injonction à tendre l'autre joue serait selon eux une maxime strictement éthique et nullement destinée à la politique. Mais l'audace de Gandhi, et le courage de la non-violence, c'est précisément de politiser cette maxime. Cette 
politisation présente un double intérêt : elle permet d'une part de réintroduire un peu d'éthique dans l'univers froid et cynique de la politique, d'autre part de tester une méthode innovante de résolution des conflits: l'action non-violente. Précisons enfin, contre ce qui est souvent dit, qu'il n'y pas d'autodestruction dans le geste de présenter la seconde joue. Le masochisme, c'eut été de tendre la même joue.

Gandhi, bien qu'il considère que le plus exigeant des courages est celui de la non-violence, sait reconnaître la présence d'une certaine forme de bravoure dans la violence. Il a souvent répété que « là où le choix existe seulement entre la lâcheté et la violence, il faut se décider pour la solution violente $\aleph^{32}$. Car, alors qu'il est impossible de voir un lâche devenir non-violent, cet espoir n'est pas interdit à un homme violent ${ }^{33}$. Celui qui défend la justice par les armes possède en effet, contrairement à celui qui s'enfuie, une vertu nécessaire pour pouvoir espérer devenir un jour non-violent : le courage. En affirmant que la violence, du fait qu'elle est courageuse, vaut mieux que la lâcheté, Gandhi s'éloigne des pacifistes. Ces derniers, en effet, adoptent une position absolutiste qui les conduit à faire du refus de partir à la guerre une maxime morale intangible. Gandhi admettait au contraire qu'il soit nécessaire - dans des situations bien particulières - d'y participer. Ainsi le courage de la non-violence semble incompatible avec la posture pacifiste qui, à certains égards, relève d'une forme de lâcheté.

Le courage de la non-violence, comme nous l'avons dit pour celui de la vérité et celui de la liberté, se conjugue aussi au niveau intellectuel. La philosophie de la non-violence fait preuve de courage intellectuel en refusant l'idéologie de la violence légitime. Précisons tout de suite que l'idéologie de la violence légitime n'affirme évidemment pas que la violence est légitime ou qu'elle est toujours légitime. Ce qu'elle postule, et à quoi s'oppose frontalement la philosophie de la non-violence, c'est que la violence est légitime pour autant qu'elle serve une cause juste. Le défi lancé à l'intellect est de rompre avec l'idéologie de la violence qui exerce « un véritable chantage sur les citoyens en affirmant que la seule façon d'être courageux est d'être violent et en les accusant à l'avance de faire preuve de lâcheté s'ils refusent de l'être $\aleph^{34}$. Face à la question des conflits - quelle attitude adopter devant tel conflit donné ? - l'idéologie de la violence légitime élabore une alternative dualiste (agir violemment ou se retirer lâchement) et dénonce aussitôt la lâcheté, aboutissant ainsi indirectement à une légitimation de la violence. Il est vrai que face à un conflit dont nous sommes étrangers nous avons toujours à choisir entre intervenir ou nous en tenir éloigné. Mais il est faux que la seule intervention possible soit de nature violente. Quand bien même les autres protagonistes du conflit (nos adversaires et/ou nos alliés) seraient armés, rien ne nous empêche d'agir sans violence, comme l'ont montré le mouvement de décolonisation de l'Inde ou celui des droits civiques en Amérique. Mais penser les choses sous cet angle, en refusant l'alternative binaire violence/lâcheté et en introduisant la possibilité d'une action non-violente, c'est déjà faire preuve de courage intellectuel puisque par cette opération de l'esprit on prend le contrepied de la culture dominante qui n'a de cesse de produire de subtiles rhétoriques argumentatives destinées à justifier l'usage de la violence. Nous employons ici l'adjectif «subtiles » car la culture dominante ne s'affiche pas ouvertement comme culture de la violence légitime. Pourtant, force est d'admettre que les sociétés occidentales reposent sur le principe que la violence est justifiée à condition qu'elle serve la justice. Tel est le présupposé éthique des doctrines universellement admises et régulièrement invoquées de la légitime défense (article 328 du code pénal français), de la guerre juste et de la raison d'Etat. La philosophie de la non-violence, en nageant seule à contre-courant de cette 
idéologie de la violence légitime, démontre son indépendance d'esprit et une certaine forme de courage intellectuel.

\section{En guise de conclusion : Le désobéissant peut-il être un militant ? Peut-il être un révolutionnaire?}

Nous venons d'esquisser de notre mieux trois figures du courage politique : le militant œuvrant pour la vérité, le révolutionnaire luttant pour la liberté et le désobéissant agissant pour la non-violence. L'effort de conceptualisation entrepris ici n'a de sens que parce qu'il ouvre à de nouvelles problématiques. Deux questions peuvent grâce à lui être posées : le désobéissant peut-il être un militant ? Et peut-il être un révolutionnaire? Il s'agit d'interrogations bien distinctes. La première nous engage dans une confrontation des notions de non-violence et de vérité et dans une évaluation de leur compatibilité. La seconde soulève un problème crucial, bien que très rarement abordé, commun à toutes les pensées de l'émancipation : la notion de révolution est-elle pensable indépendamment de celle de violence? Nous nous contentons ici d'expliciter ces deux problématiques, dont l'élucidation mériterait bien plus que cette succincte conclusion.

Clarifier la relation entre le désobéissant et le militant revient à confronter la nonviolence à la vérité puisque la première est au premier ce que la seconde et au second : sa forme spécifique de courage. Cette confrontation pourrait être élaborée selon le prisme de la pensée gandhienne, car c'est là qu'elle est née et qu'elle demeure le mieux théorisée. La société politique est selon Gandhi l'espace de rencontre et de confrontation de différentes prétentions à la vérité; chaque prétention (claim) étant correcte mais partielle. Et les individus n'ont pas conscience de la dimension relative de leur vérité. Or l'histoire nous enseigne que celui qui croit détenir la Vérité absolue est capable d'horribles crimes pour la défendre ${ }^{35}$. L'humanité a commis les pires atrocités « au nom de la Vérité » (vérité religieuse, vérité de l'Histoire, vérité de la Race, vérité de la Science). La question est donc la suivante: comment Gandhi va-t-il pouvoir maintenir sa conception de la politique définie comme recherche de la vérité, tout en évitant une dérive autoritaire ou anarchique de l'activité politique? Comment garantir la nonviolence tout en exigeant la recherche de la vérité ?

De deux manières. Premièrement, par une distinction épistémologique entre Vérité absolue et vérités relatives : la Vérité absolue existe, mais les hommes sont incapables d'y accéder ; puisqu'ils ne peuvent connaître que des vérités relatives, les individus n'ont pas le droit de juger autrui, et encore moins de le punir de façon violente. Deuxièmement, pour que la quête politique de la Vérité demeure non-violente, il faut nécessairement que la Vérité et la non-violence ne fasse qu'un. Jean-Marie Muller détaille ce second point: "L'histoire est là pour attester - et l'expérience le confirme tous les jours - que la vérité devient un vecteur de violence dès lors qu'elle s'absolutise et n'est pas ancrée dans l'exigence de non-violence. Si la vérité n'implique pas par elle-même la délégitimation radicale de la violence, alors viendra toujours un moment où la violence apparaitra naturellement comme un moyen légitime pour défendre la vérité $\aleph^{36}$. Gandhi, conscient de cette exigence, avait solidement lié la non-violence à la vérité de manière que cette dernière ne s'achève pas en conflit armé. L'une et l'autre sont indispensables pour « former un tout unique. C'est pourquoi, parfois, on les compare aux deux faces d'une même pièce de monnaie. L'une est 
indissociable de l'autre. La valeur de la pièce est la même quel que soit le côté qu'on regarde $»^{37}$.

Le désobéissant peut-il être révolutionnaire? Cette seconde question invite à (re)conceptualiser la notion de révolution non-violente. Le citoyen ordinaire comme le chercheur en sciences sociales associent instinctivement "violence » et "révolution ». Cette évidence demande pourtant à être questionnée. Le fait que, lors des quarante dernières années, cinquante des soixante-sept renversements de régimes autoritaires aient abouti grâce à la résistance civile non-violente impose de redéfinir la notion de révolution en la détachant de celle de violence. Etant entendu que tout ou presque a déjà été dit et expérimenté du côté de la version "violente » de la révolution, une révolution non-violente est-elle historiquement possible, théoriquement concevable et, le cas échéant, quel sens donner à cette notion et quelles sont les caractéristiques de ce phénomène ? Ces préoccupations permettraient selon nous d'alimenter le renouveau de la philosophie politique contemporaine ${ }^{38}$.

\section{BIBLIOGRAPHIE}

ABENSOUR Miguel (2009), Pour une philosophie politique critique, Paris, Sens \& Tonka

ARENDT Hannah (1972), « La désobéissance civile », Du mensonge à la violence, Paris, Calmann-Lévy

ARENDT Hannah (1995), Qu'est-ce que la politique ?, Paris, Seuil

CAMUS, Albert, L'homme révolté, Paris, Gallimard, 1951

FOUCAULT Michel (décembre 2008), « Le courage de la vérité : l'ascète, le révolutionnaire et

l'artiste », in Esprit, Paris, pp. 51-60

GANDHI (1960), Lettre à l'ashram, Paris, Albin Michel

GANDHI (1990), Tous les hommes sont frères, Paris, Gallimard

GANDHI (2007), Résistance non-violente, Paris, Buchet/Chastel

GROS Frédéric (2006), Etats de violence, Essai sur la fin de la guerre, Paris, Gallimard, 2006

GUERIN Daniel (1945), Fascisme et Grand capital, Paris, Gallimard

HADOT Pierre (1995), Qu'est-ce que la philosophie antique ?, Paris, Gallimard

MULLER Jean-Marie (1994), Gandhi, la sagesse de la non-violence, Paris, Desclée de Brouwer

MULLER Jean-Marie (2005), Dictionnaire de la non-violence, Gordes, Les Editions du Relié

MULLER Jean-Marie (2008), Le courage de la non-violence, Paris, Les Editions du Relié

ORWELL Georges (1982), Hommage à la Catalogne, Paris, 10/18

THOREAU Henry David (2007), La désobéissance civile, Paris, Le passager clandestin

WEIL Simone (1960), « Réflexions pour déplaire », Ecrits historiques et politiques, Paris, Gallimard

ZINN Howard (2006), L'impossible neutralité, Marseille, Agone 


\section{NOTES}

1. Si nous ne distinguons pas le «sujet politique» du "citoyen " c'est que, à distance de l'approche juridico-libérale, nous refusons d'envisager la citoyenneté comme un statut juridique. Est citoyen celui qui agit. La citoyenneté n'est jamais acquise ni donné mais revient à celui qui s'engage publiquement. A ce propos on lira ISIN, Engin, « Theorizing Acts of Citizenship », in Acts of Citizenship, Zed Books, London, 2008, pp. 15-43

2. THOREAU, Henry David, La désobéissance civile, Paris, Le passager clandestin, 2007, p. 27

3. ZINN, Howard, L'impossible neutralité, Marseille, Agone, 2006, p. 14. À ce sujet Guy Béart chantait déjà : «Le premier qui dit la vérité se trouve toujours sacrifié // Le premier qui dit la vérité il doit être exécuté ».

4. Il faut aussi garder à l'esprit que certaines paroles, en vertu de leur dimension performative, sont en elles-mêmes des actes.

5. GROS, Frédéric, Etats de violence, Essai sur la fin de la guerre, Paris, Gallimard, 2006, p. 22

6. Ibid., p. 24

7. FOUCAULT, Michel, "Le courage de la vérité : l'ascète, le révolutionnaire et l'artiste », in Esprit, Paris, décembre 2008, pp. 51-60.

8. Pour une étude des mouvements chrétiens millénaristes du Moyen-Age, donnant corps à la vérité du Christ à travers leurs pratiques ascétiques et mystiques, on se référera à l'excellent ouvrage de COHN, Norman, Les Fanatiques de l'Apocalypse, Paris, Julliard, 1962.

9. GUERIN, Daniel, Fascisme et Grand capital, Paris, Gallimard, 1945

10. WEIL, Simone, «Réflexions pour déplaire », Ecrits historiques et politiques, Gallimard, Paris, 1960, pp. 218-219

11. WEIL, Simone, Note sur la suppression générale des partis politiques, Paris, Climats, 2007, p. 69

12. Ibid., p. 60

13. ARENDT, Hannah, Qu'est-ce que la politique ?, Paris, Seuil, 1995, p. 83

14. Ibid.

15. Nous suivons ici le travail de Miguel Abensour sur l'héroïsme révolutionnaire de Arendt. Voir ABENSOUR, Miguel, Pour une philosophie politique critique, Paris, Sens \& Tonka, 2009, pp. 257-263

16. Ibid., p. 257

17. Ibid., p. 262

18. CAMUS, Albert, L'homme révolté, Paris, Gallimard, 1951, p. 259

19. GROS, op. cit., p. 43

20. HADOT, Pierre, Qu'est-ce que la philosophie antique ?, Paris, Gallimard, 1995, p. 367

21. Partido Obrero de Unificación Marxista, de tendance trotskiste.

22. ORWELL, Georges, Hommage à la Catalogne, Paris, 10/18, 1982, pp. 12-13

23. Ibid., p. 15

24. Ibid., p. 111

25. GANDHI, Résistance non-violente, Paris, Buchet/Chastel, 2007, p. 140

26. GANDHI, Tous les hommes sont frères, Paris, Gallimard, 1990, p. 162

27. MULLER, Jean-Marie, Gandhi, la sagesse de la non-violence, Paris, Desclée de Brouwer, 1994, pp. 33-34

28. GANDHI, cité in MULLER, Gandhi, p. 34

29. GANDHI, Lettre à l'ashram, Paris, Albin Michel, 1960, p. 110

30. Bakounine pensait par ailleurs qu'il valait mieux faire appel à la conscience de l'individu qu'à sa raison. Car la raison peut devenir un obstacle à l'action: elle entraîne la division interne et l'inhabilité à agir. L'analyse rationnelle, parce qu'elle est sans fin, paralyse l'action. La 
conscience, au contraire, se préoccupe davantage de mener une action efficace que d'atteindre une pensée pure.

31. ARENDT, Hannah, «La désobéissance civile », Du mensonge à la violence, Paris, Calmann-Lévy, 1972, p. 69

32. GANDHI, Tous les hommes sont frères, p. 182

33. Ibid. p. 179

34. MULLER, Jean-Marie, Dictionnaire de la non-violence, Gordes, Les Editions du Relié, 2005, p. 77

35. Hans Kelsen nous avait averti sur le lien entre une épistémologie de la vérité et l'autocratie d'une part, et entre une épistémologie positiviste-relativiste et la démocratie d'autre part. Gandhi, à la même époque, avait perçu les risques de violence inhérents à une politique fondée sur une vérité substantielle. Celui qui pense que la Vérité est Dieu est amené à la défendre à tout prix contre l'erreur et se fait un devoir de combattre les hérétiques par tous les moyens, y compris la violence. Le risque est donc important que la recherche de la vérité aboutisse en guerre sainte des temps modernes. L'histoire, écrit Gandhi, nous enseigne que des "milliers d'atrocités sans nom ont été commises au nom de Dieu ». Mais alors que certains bourreaux justifiaient ces atrocités par la défense de la soi-disante vérité, Gandhi assurait ne pas connaitre " de plus grand péché que d'opprimer l'innocent au nom de la vérité ».

36. MULLER, Jean-Marie, Le courage de la non-violence, Paris, Les Editions du Relié, 2008, p. 139

37. GANDHI, Tous les hommes sont frères, p. 151. De même que la vérité est organiquement liée à la non-violence, le mensonge est lié à la violence. Ainsi, dans la controverse opposant Kant à Constant sur le droit de mentir, Gandhi se situerait davantage aux côtés du philosophe de Königsberg.

38. Qu'il nous soit permis à ce sujet de renvoyer à notre article «Vers une théorie de la révolution non-violente" (Dissensus, ${ }^{\circ} 4$, avril 2011, pp. 164-184), dans lequel nous esquissons une histoire de la notion de révolution non-violente.

\section{RÉSUMÉS}

La question des modalités de la subjectivation politique - comment produire de la citoyenneté ? invite à réinvestir la notion de courage, entendu comme ce qui fait passer un individu de l'intelligence d'un problème à la volonté de le résoudre. Le sujet politique fait preuve de courage en sortant des coulisses pour venir s'exposer sur la scène politique. Mais, cette définition générale étant admise, force est de constater que la notion n'est pas monolithique. En effet, la modernité politique, tant au niveau de la pratique que des idées, nous permet de dresser une typologie tripartite des différentes formes de courage : le courage de la vérité, qu'à l'instar de Foucault nous nommerons parrhèsia; le courage de la liberté, qu'à l'instar d'Arendt nous nommerons héroïsme; et le courage de la non-violence, qu'à l'instar de Gandhi nous nommerons satyagraha. La parrhèsia est le courage de dire le vrai dans un monde de mensonges et de «faire de sa vie un témoignage de vérité ». L'héroïsme est le courage de préférer la mort à la servitude au sens où, comme le disait le révolutionnaire mexicain Emiliano Zapata, «mieux vaut mourir debout que de vivre à genoux ! " Enfin, le satyagraha est le courage de « combattre le mal tout en refusant d'imiter sa violence ». 
INDEX

Mots-clés : courage, subjectivation politique, parrhèsia, héroïsme, satyagraha, critique du travail, correspondance, artistes, socialisme vert, travailleurs culturels, Wertkritik, Gorz André, dialogue, Vincent Jean-Marie, écologie, modèles alternatifs, Marx Karl, penser le Capital, ethos du travail, Eglise Orthodoxe, salariat, domination

\section{AUTEUR}

\section{MANUEL CERVERA-MARZAL}

Diplômé de Sciences-Po Paris et du Master « Sociologie et Philosophie politique » de l'Université Paris-Diderot. Travaille sur la désobéissance civile et l'action directe non-violente. Doctorant en co-tutelle à l'Université Libre de Bruxelles (sous la direction de Justine Lacroix) et à l'Université Paris-Diderot (sous la direction d'Etienne Tassin). 